\title{
A POMDP Framework for Coordinated Guidance of Autonomous UAVs for Multitarget Tracking
}

\author{
Scott A. Miller, ${ }^{1}$ Zachary A. Harris, ${ }^{1}$ and Edwin K. P. Chong ${ }^{2}$ \\ ${ }^{1}$ Numerica Corporation, 4850 Hahns Peak Drive, Suite 200, Loveland, CO 80538, USA \\ ${ }^{2}$ Department of Electrical and Computer Engineering (ECE), Colorado State University, Fort Collins, \\ CO 80523-1373, USA
}

Correspondence should be addressed to Scott A. Miller, scott.miller@numerica.us

Received 1 August 2008; Accepted 1 December 2008

Recommended by Matthijs Spaan

This paper discusses the application of the theory of partially observable Markov decision processes (POMDPs) to the design of guidance algorithms for controlling the motion of unmanned aerial vehicles (UAVs) with onboard sensors to improve tracking of multiple ground targets. While POMDP problems are intractable to solve exactly, principled approximation methods can be devised based on the theory that characterizes optimal solutions. A new approximation method called nominal belief-state optimization (NBO), combined with other application-specific approximations and techniques within the POMDP framework, produces a practical design that coordinates the UAVs to achieve good long-term mean-squared-error tracking performance in the presence of occlusions and dynamic constraints. The flexibility of the design is demonstrated by extending the objective to reduce the probability of a track swap in ambiguous situations.

Copyright (C) 2009 Scott A. Miller et al. This is an open access article distributed under the Creative Commons Attribution License, which permits unrestricted use, distribution, and reproduction in any medium, provided the original work is properly cited.

\section{Introduction}

Interest in unmanned aerial vehicles (UAVs) for applications such as surveillance, search, and target tracking has increased in recent years, owing to significant progress in their development and a number of recognized advantages in their use $[1,2]$. Of particular interest to this special issue is the interplay among signal processing, robotics, and automatic control in the success of UAV systems.

This paper describes a principled framework for designing a planning and coordination algorithm to control a fleet of UAVs for the purpose of tracking ground targets. The algorithm runs on a central fusion node that collects measurements generated by sensors onboard the UAVs, constructs tracks from those measurements, plans the future motion of the UAVs to maximize tracking performance, and sends motion commands back to the UAVs based on the plan.

The focus of this paper is to illustrate a design framework based on the theory of partially observable Markov decision processes (POMDPs), and to discuss practical issues related to the use of the framework. With this in mind, the problem scenarios presented here are idealized, and are meant to illustrate qualitative behavior of a guidance system design. Moreover, the particular approximations employed in the design are examples and can certainly be improved. Nevertheless, the intent is to present a design approach that is flexible enough to admit refinements to models, objectives, and approximation methods without damaging the underlying structure of the framework.

Section 2 describes the nature of the UAV guidance problem addressed here in more detail, and places it in the context of the sensor resource management literature. The detailed problem specification is presented in Section 3, and our method for approximating the solution is discussed in Section 4. Several features of our approach are already apparent in the case of a single UAV, as discussed in Section 5. The method is extended to multiple UAVs in Section 6, where coordination of multiple sensors is demonstrated. In Section 7, we illustrate the flexibility of the POMDP framework by modifying it to include more complex tracking objectives such as preventing track swaps. Finally, we conclude in Section 8 with summary remarks and future directions. 


\section{Problem Description}

The class of problems we pose in this paper is a rather schematic representation of the UAV guidance problem. Simplifications are assumed for ease of presentation and understanding of the key issues involved in sensor coordination. These simplifications include the following.

2-D Motion. The targets are assumed to move in a plane on the ground, while the UAVs are assumed to fly at a constant altitude above the ground.

Position Measurements. The measurements generated by the sensors are 2-D position measurements with associated covariances describing the position uncertainty. A simplified visual sensor (camera plus image processing) is assumed, which implies that the angular resolution is much better than the range resolution.

Perfect Tracker. We assume that there are no false alarms and no missed detections, so exactly one measurement is generated for each target visible to the sensor. Also, perfect data association is usually assumed, so the tracker knows which measurement came from which target, though this assumption is relaxed in Section 7 when track ambiguity is considered.

Nevertheless, the problem class has a number of important features that influence the design of a good planning algorithm. These include the following.

Dynamic Constraints. These appear in the form of constraints on the motion of the UAVs. Specifically, the UAVs fly at a constant speed and have bounded lateral acceleration in the plane, which limits their turning radius. This is a reasonable model of the characteristics of small fixed-wing aircraft. The presence of dynamic constraints implies that the planning algorithm needs to include some form of lookahead for good long-term performance.

Randomness. The measurements have random errors, and the models of target motion are random as well. However, in most of our simulations the actual target motion is not random.

Spatially Varying Measurement Error. The range error of the sensor is an affine function of the distance between the sensor and the target. The bearing error of the sensor is constant, but that translates to a proportional error in Cartesian space as well. This spatially varying error is what makes the sensor placement problem meaningful.

Occlusions. There are occlusions in the plane that block the visibility of targets from sensors when they are on opposite sides of an occlusion. The occlusions are generally collections of rectangles in our models, though in the case studies presented they appear more as walls (thin rectangles). Targets are allowed to cross occlusions, and of course the UAVs are allowed to fly over them; their purpose is only to make the observation of targets more challenging.

Tracking Objectives. The performance objectives considered here are related to maintaining the best tracks on the targets. Normally, that means minimizing the mean-squared error between tracks and targets, but in Section 7 we also consider the avoidance of track swaps as a performance objective. This differs from most of the guidance literature, where the objective is usually posed as interpolation of way-points.

In Section 3 we demonstrate that the UAV guidance problem described here is a POMDP. One implication is that the exact problem is in general formally undecidable [3], so one must resort to approximations. However, another implication is that the optimal solution to this problem is characterized by a form of Bellman's principle, and this principle can be used as a basis for a structured approximation of the optimal solution. In fact, the main goal of this paper is to demonstrate that the design of the UAV guidance system can be made practical by a limited and precisely understood use of heuristics to approximate the ideal solution. That is, the heuristics are used in such a way that their influence may be relaxed and the solution improved as more computational resources become available.

The UAV guidance problem considered here falls within the class of problems known as sensor resource management [4]. In its full generality, sensor resource management encompasses a large body of problems arising from the increasing variety and complexity of sensor systems, including dynamic tasking of sensors, dynamic sensor placement, control of sensing modalities (such as waveforms), communication resource allocation, and task scheduling within a sensor [5]. A number of approaches have been proposed to address the design of algorithms for sensor resource management, which can be broadly divided into two categories: myopic and nonmyopic.

Myopic approaches do not explicitly account for the future effects of sensor resource management decisions (i.e., there is no explicit planning or "lookahead"). One approach within this category is based on fuzzy logic and expert systems [6], which exploits operator knowledge to design a resource manager. Another approach uses informationtheoretic measures as a basis for sensor resource management [7-9]. In this approach, sensor controls are determined based on maximizing a measure of "information."

Nonmyopic approaches to sensor resource management have gained increasing interest because of the need to account for the kinds of requirements described in this paper, which imply that foresight and planning are crucial for good long-term performance. In the context of UAV coordination and control, such approaches include the use of guidance rules [2,10-12], oscillator models [13], and information-driven coordination $[1,14]$. A more general approach to dealing with nonmyopic resource management involves stochastic dynamic programming formulations of the problem (or, more specifically, POMDPs). As pointed out in Section 4, exact optimal solutions are practically infeasible to compute. Therefore, recent effort has focused on obtaining 
approximate solutions, and a number of methods have been developed (e.g., see [15-20]). This paper contributes to the further development of this thrust by introducing a new approximation method, called nominal belief-state optimization, and applying it to the UAV guidance problem.

Approximation methods for POMDPs have been prominent in the recent literature on artificial intelligence (AI), under the rubric of probabilistic robotics [21]. In contrast to much of the POMDP methods in the AI literature, a unique feature of our current approach is that the state and action spaces in our UAV guidance problem formulation is continuous. We should note that some recent AI efforts have also treated the continuous case (e.g., see [22-24]), though in different settings.

\section{POMDP Specification and Solution}

In this section, we describe the mathematical formulation of our guidance problem as a partially observable Markov decision process (POMDP). We first provide a general definition of POMDPs. We provide this background exposition for the sake of completeness-readers who already have this background can skip this subsection. Then, we proceed to the specification of the POMDP for the guidance problem. Finally, we discuss the nature of POMDP solutions, leading up to a discussion of approximation methods in the next section. For a full treatment of POMDPs and related background, see [25]. For a discussion of POMDPs in sensor management, see [5].

3.1. Definition of POMDP. A POMDP is a controlled dynamical process, useful in modeling a wide range of resource control problems. To specify a POMDP model, we need to specify the following components:

(i) a set of states (the state space) and a distribution specifying the random initial state;

(ii) a set of possible actions;

(iii) a state-transition law specifying the next-state distribution given an action taken at a current state;

(iv) a set of possible observations;

(v) an observation law specifying the distribution of observations depending on the current state and possibly the action;

(vi) a cost function specifying the cost (real number) of being in a given state and taking a given action.

In the next subsection, we specify these components for our guidance problem.

As a POMDP evolves over time as a dynamical process, we do not have direct access to the states. Instead, all we have are the observations generated over time, providing us with clues of the actual underlying states (hence the term partially observable). These observations might, in some cases, allow us to infer exactly what states actually occurred. However, in general, there will be some uncertainty in our knowledge of the states. This uncertainty is represented by the belief state, which is the a posteriori distribution of the underlying state given the history of observations. The belief states summarize the "feedback" information that is needed for controlling the system. Conveniently, the belief state can easily be tracked over time using Bayesian methods. Indeed, as pointed out below, in our guidance problem the belief state is a quantity that is already available (approximately) as track states.

Once we have specified the above components of a POMDP, the guidance problem is posed as an optimization problem where the expected cumulative cost over a time horizon is the objective function to be minimized. The decision variables in this optimization problem are the actions to be applied over the planning horizon. However, because of the stochastic nature of the problem, the optimal actions are not fixed but are allowed to depend on the particular realization of the random variables observed in the past. Hence, the optimal solution is a feedback-control rule, usually called a policy. More formally, a policy is a mapping that, at each time, takes the belief state and gives us a particular control action, chosen from the set of possible actions. What we seek is an optimal policy. We will characterize optimal policies in a later subsection, after we discuss the POMDP formulation of the guidance problem.

3.2. POMDP Formulation of Guidance Problem. To formulate our guidance problem in the POMDP framework, we must specify each of the above components as they relate to the guidance system. This subsection is devoted to this specification.

States. In the guidance problem, three subsystems must be accounted for in specifying the state of the system: the sensor(s), the target(s), and the tracker. More precisely, the state at time $k$ is given by $x_{k}=\left(s_{k}, \zeta_{k}, \xi_{k}, P_{k}\right)$, where $s_{k}$ represents the sensor state, $\zeta_{k}$ represents the target state, and $\left(\xi_{k}, P_{k}\right)$ represents the track state. The sensor state $s_{k}$ specifies the locations and velocities of the sensors (UAVs) at time $k$. The target state $\zeta_{k}$ specifies the locations, velocities, and accelerations of the targets at time $k$. Finally, the track state $\left(\xi_{k}, P_{k}\right)$ represents the state of the tracking algorithm; $\xi_{k}$ is the posterior mean vector and $P_{k}$ is the posterior covariance matrix, standard in Kalman filtering algorithms. The representation of the state into a vector of state variables is an instance of a factored model [26].

Action. In our guidance problem, we assume a standard model where each UAV flies at constant speed and its motion is controlled through turning controls that specify lateral instantaneous accelerations. The lateral accelerations can take values in an interval $\left[-a_{\max }, a_{\max }\right]$, where $a_{\max }$ represents a maximum limit on the possible lateral acceleration. So, the action at time $k$ is given by $a_{k} \in[-1,1]^{N_{\text {sens }}}$, where $N_{\text {sens }}$ is the number of UAVs, and the components of the vector $a_{k}$ specify the normalized lateral acceleration of each UAV.

State-Transition Law. The state-transition law specifies how each component of the state changes from one-time step to 
the next. In general, the transition law takes the following form:

$$
x_{k+1} \sim p_{k}\left(\cdot \mid x_{k}\right)
$$

for some time-varying distribution $p_{k}$. However, the model for the UAV guidance problem constrains the form of the state transition law. The sensor state evolves according to

$$
s_{k+1}=\psi\left(s_{k}, a_{k}\right),
$$

where $\psi$ is the map that defines how the state changes from one-time step to the next depending on the acceleration control as described above. The target state evolves according to

$$
\zeta_{k+1}=f\left(\zeta_{k}\right)+v_{k}
$$

where $v_{k}$ represents an i.i.d. random sequence and $f$ represents the target motion model. Most of our simulation results use a nearly constant velocity (NCV) target motion model, except for Section 6.2 which uses a nearly constant acceleration (NCA) model. In all cases $f$ is linear, and $v_{k}$ is normally distributed. We write $v_{k} \sim \mathcal{N}\left(0, Q_{k}\right)$ to indicate the noise is normal with zero mean and covariance $Q_{k}$.

Finally, the track state $\left(\xi_{k}, P_{k}\right)$ evolves according to a tracking algorithm, which is defined by a data association method and the Kalman filter update equations. Since our focus is on UAV guidance and not on practical tracking issues, in most cases a "truth tracker" is used, which always associates a measurement with the track corresponding to the target being detected. Only in Section 7 is a nonideal data association considered, for the purpose of evaluating performance with ambiguous associations.

Observations and Observation Law. In general, the observation law takes the following form:

$$
z_{k} \sim q_{k}\left(\cdot \mid x_{k}\right)
$$

for some time-varying distribution $q_{k}$. In our guidance problem, since the state has four separate components, it is convenient to express the observation with four corresponding components (a factored representation). The sensor state and track state are assumed to be fully observable. So, for these components of the state, the observations are equal to the underlying state components:

$$
z_{k}^{s}=s_{k}, \quad z_{k}^{\xi}=\xi_{k}, \quad z_{k}^{P}=P_{k} .
$$

The target state, however, is not directly observable; instead, what we have are random measurements of the target state that are functions of the locations of the targets and the sensors.

Let $\zeta_{k}^{\text {pos }}$ and $s_{k}^{\text {pos }}$ represent the position vectors of the target and sensor, respectively, and let $h\left(\zeta_{k}, s_{k}\right)$ be a booleanvalued function that is true if the line of sight from $s_{k}^{\text {pos }}$ to $\zeta_{k}^{\text {pos }}$ is unobscured by any occlusions. Furthermore, we define a $2 \mathrm{D}$ position covariance matrix $R_{k}\left(\zeta_{k}, s_{k}\right)$ that reflects a $10 \%$ uncertainty in the range from sensor to target, and $0.01 \pi$ radian angular uncertainty, where the range is taken to be at least 10 meters. Then, the measurement of the target state at time $k$ is given by

$$
z_{k}^{\zeta}= \begin{cases}\zeta_{k}^{\text {pos }}+w_{k}, & \text { if } h\left(\zeta_{k}, s_{k}\right)=\text { true } \\ \varnothing(\text { no measurement }), & \text { if } h\left(\zeta_{k}, s_{k}\right)=\text { false }\end{cases}
$$

where $w_{k}$ represents an i.i.d. sequence of noise values distributed according to the normal distribution $\mathcal{N}\left(0, R_{k}\left(\zeta_{k}\right.\right.$, $\left.s_{k}\right)$.

Cost Function. The cost function we most commonly use in our guidance problem is the mean-squared tracking error, defined by the following:

$$
C\left(x_{k}, a_{k}\right)=\mathrm{E}_{v_{k}, w_{k+1}}\left[\left\|\zeta_{k+1}-\xi_{k+1}\right\|^{2} \mid x_{k}, a_{k}\right] .
$$

In Section 7.1, we describe a different cost function which we use for detecting track ambiguity.

Belief State. Although not a part of the POMDP specification, it is convenient at this point to define our notation for the belief state for the guidance problem. The belief state at time $k$ is given by the following:

$$
b_{k}=\left(b_{k}^{s}, b_{k}^{\zeta}, b_{k}^{\xi}, b_{k}^{P}\right)
$$

where

$$
\begin{gathered}
b_{k}^{s}(s)=\delta\left(s-s_{k}\right), \\
b_{k}^{\zeta} \text { updated with } z_{k}^{\zeta} \text { using Bayes theorem } \\
b_{k}^{\xi}(\xi)=\delta\left(\xi-\xi_{k}\right), \\
b_{k}^{P}(P)=\delta\left(P-P_{k}\right) .
\end{gathered}
$$

Note that those components of the state that are directly observable have delta functions representing their corresponding belief-state components.

We have deliberately distinguished between the belief state and the track state (the internal state of the tracker). The reason for this distinction is so that the model is general enough to accommodate a variety of tracking algorithms, even those that are acknowledged to be severe approximations of the actual belief state. For the purpose of control, it is natural to use the internal state of the tracker as one of the inputs to the controller (and it is intuitive that the control performance would benefit from the use of this information). Therefore, it is appropriate to incorporate the track state into the the POMDP state space, even if this is not prima facie obvious.

3.3. Optimal Policy. Given the POMDP formulation of our problem, our goal is to select actions over time to minimize the expected cumulative cost (we take expectation here because the cumulative cost is a random variable, being a function of the random evolution of $x_{k}$ ). To be specific, suppose we are interested in the expected cumulative cost over a time horizon of length $H: k=0,1, \ldots, H-1$. 
The problem is to minimize the cumulative cost over horizon $H$, given by the following:

$$
J_{H}=\mathrm{E}\left[\sum_{k=0}^{H-1} C\left(x_{k}, a_{k}\right)\right] .
$$

The goal is to pick the actions so that the objective function is minimized. In general, the action chosen at each time should be allowed to depend on the entire history up to that time (i.e., the action at time $k$ is a random variable that is a function of all observable quantities up to time $k$ ). However, it turns out that if an optimal choice of such a sequence of actions exists, then there is an optimal choice of actions that depends only on "belief-state feedback." In other words, it suffices for the action at time $k$ to depend only on the belief state at time $k$, as alluded to before.

Let $b_{k}$ be the belief state at time $k$, which is a distribution over states,

$$
b_{k}(x)=\mathrm{P}_{x_{k}}\left(x \mid z_{0}, \ldots, z_{k} ; a_{0}, \ldots, a_{k-1}\right)
$$

updated incrementally using Bayes rule. The objective can be written in terms of belief states

$$
J_{H}=\mathrm{E}\left[\sum_{k=0}^{H-1} c\left(b_{k}, a_{k}\right) \mid b_{0}\right], \quad c(b, a)=\int C(x, a) b(x) d x,
$$

where $\mathrm{E}\left[\cdot \mid b_{o}\right]$ represents conditional expectation given $b_{0}$. Let $\mathcal{B}$ represent the set of possible belief states, and let $\mathcal{A}$ represent the set of possible actions. So what we seek is, at each time $k$, a mapping $\pi_{k}^{*}: \mathscr{B} \rightarrow \mathcal{A}$ such that if we perform action $a_{k}=\pi_{k}^{*}\left(b_{k}\right)$, then the resulting objective function is minimized. This is the desired optimal policy.

The key result in POMDP theory is Bellman's principle. Let $J_{H}^{*}\left(b_{0}\right)$ be the optimal objective function value (over horizon $H$ ) with $b_{0}$ as the initial belief state. Then, Bellman's principle states that

$$
\pi_{0}^{*}\left(b_{0}\right)=\underset{a}{\operatorname{argmin}}\left\{c\left(b_{0}, a\right)+\mathrm{E}\left[J_{H-1}^{*}\left(b_{1}\right) \mid b_{0}, a\right]\right\}
$$

is an optimal policy, where $b_{1}$ is the random next belief state (with distribution depending on $a$ ), $\mathrm{E}\left[\cdot \mid b_{0}, a\right]$ represents conditional expectation (given $b_{0}$ and action $a$ ) with respect to the random next state $b_{1}$, and $J_{H-1}^{*}\left(b_{1}\right)$ is the optimal cumulative cost over the time horizon $1, \ldots, H$ starting with belief state $b_{1}$.

Define the $Q$-value of taking action $a$ at state $b_{0}$ as follows:

$$
Q_{H}\left(b_{0}, a\right)=c\left(b_{0}, a\right)+\mathrm{E}\left[J_{H-1}^{*}\left(b_{1}\right) \mid b_{0}, a\right] .
$$

Then, Bellman's principle can be rewritten as follows:

$$
\pi_{0}^{*}\left(b_{0}\right)=\underset{a}{\operatorname{argmin}} Q_{H}\left(b_{0}, a\right),
$$

that is, the optimal action at belief state $b_{0}$ is the one with smallest $Q$-value at that belief state. Thus, Bellman's principle instructs us to minimize a modified cost function $\left(Q_{H}\right)$ that includes the term $\mathrm{E}\left[J_{H-1}^{*}\right]$ indicating the expected future cost of an action; this term is called the expected cost-togo (ECTG). By minimizing the $Q$-value that includes the ECTG, the resulting policy has a lookahead property that is a common theme among POMDP solution approaches.

For the optimal action at the next belief state $b_{1}$, we would similarly define the $Q$-value

$$
Q_{H-1}\left(b_{1}, a\right)=c\left(b_{1}, a\right)+\mathrm{E}\left[J_{H-2}^{*}\left(b_{2}\right) \mid b_{1}, a\right],
$$

where $b_{2}$ is the random next belief state and $J_{H-2}^{*}\left(b_{2}\right)$ is the optimal cumulative cost over the time horizon $2, \ldots, H$ starting with belief state $b_{2}$. Bellman's principle then states that the optimal action is given by the following:

$$
\pi_{1}^{*}\left(b_{1}\right)=\underset{a}{\operatorname{argmin}} Q_{H-1}\left(b_{1}, a\right) .
$$

A common approach in online optimization-based control is to assume that the horizon is long enough that the difference between $Q_{H}$ and $Q_{H-1}$ is negligible. This has two implications: first, the time-varying optimal policy $\pi_{k}^{*}$ may be approximated by a stationary policy, denoted $\pi^{*}$; second, the optimal policy is given by the following:

$$
\pi^{*}(b)=\underset{a}{\operatorname{argmin}} Q_{H}(b, a),
$$

where now the horizon is fixed at $H$ regardless of the current time $k$. This approach is called receding horizon control, and is practically appealing because it provides lookahead capability without the technical difficulty of infinite-horizon control. Moreover, there is usually a practical limit to how far models may be usefully predicted. Henceforth, we will assume the horizon length is constant and drop it from our notation.

In summary, we seek a policy $\pi^{*}(b)$ that, for a given belief state $b$, returns the action $a$ that minimizes $Q(b, a)$, which in the receding-horizon case is

$$
Q(b, a)=c(b, a)+\mathrm{E}\left[J^{*}\left(b^{\prime}\right) \mid b, a\right]
$$

where $b^{\prime}$ is the (random) belief state after applying action $a$ at belief state $b$, and $c(b, a)$ is the associated cost. The second term in the $Q$-value is in general difficult to obtain, especially because the belief-state space is large. For this reason, approximation methods are necessary. In the next section, we describe our algorithm for approximating $\operatorname{argmin}_{a} Q(b, a)$.

We should re-emphasize here that the action space in our UAV guidance problem is a hypercube, which is a continuous space of possible actions. The optimization involved in performing $\operatorname{argmin}_{a} Q(b, a)$ therefore involves a search algorithm over this hypercube. Our focus in this paper is on a new method to approximate $Q(b, a)$ and not on how to minimize it. Therefore, in this paper we simply use a generic search method to perform the minimization. More specifically, in our simulation studies, we used Matlab's fmincon function. We should point out that in related work, other authors have considered the problem of designing a good search algorithm (e.g., [27]). 


\section{Approximation Method}

There are two aspects of a general POMDP that make it intractable to solve exactly. First, it is a stochastic control problem, so the dynamics are properly understood as constraints on distributions over the state space, which are infinite dimensional in the case of a continuous state space as in our tracking application. In practice, solution methods for Markov decision processes employ some parametric representation or nonparametric (i.e., Monte Carlo or "particle") representation of the distribution, to reduce the problem to a finite-dimensional one. Intelligent choices of finitedimensional approximations are derived from Bellman's principle characterizing the optimal solution. POMDPs, however, have the additional complication that the state space itself is infinite dimensional, since it includes the belief state which is a distribution; hence, the belief state must also be approximated by some finite-dimensional representation. In Section 4.1, we present a finite-dimensional approximation to the problem called nominal belief-state optimization (NBO), which takes advantage of the particular structure of the tracking objective in our application.

Secondly, in the interest of long-term performance, the objective of a POMDP is often stated over an arbitrarily long or infinite horizon. This difficulty is typically addressed by truncating the horizon to a finite length, the effect of which is discussed in Section 4.2.

Before proceeding to the detailed description of our NBO approach, we first make two simplifying approximations that follow from standard assumptions for tracking problems. The first approximation, which follows from the assumption of a correct tracking model and Gaussian statistics, is that the belief-state component for the target can be expressed as follows:

$$
b_{k}^{\zeta}(\zeta)=\mathcal{N}\left(\zeta-\xi_{k}, P_{k}\right)
$$

and can be updated using (extended) Kalman filtering. We adopt this approximation for the remainder of this paper. The second approximation, which follows from the additional assumption of correct data association, is that the cost function can be written as follows:

$$
\begin{aligned}
c\left(b_{k}, a_{k}\right) & =\int \mathrm{E}_{v_{k}, w_{k+1}}\left[\left\|\zeta_{k+1}-\xi_{k+1}\right\|^{2} \mid s_{k}, \zeta, \xi_{k}, a_{k}\right] b_{k}^{\zeta}(\zeta) d \zeta \\
& =\operatorname{Tr} P_{k+1} .
\end{aligned}
$$

In Section 7, we study the impact of this approximation in the context of tracking with data association ambiguity (i.e., when we do not necessarily have the correct data association), and consider a different cost function that explicitly takes into account the data association ambiguity.

4.1. Nominal Belief-State Optimization (NBO). A number of POMDP approximation methods have been studied in the literature. It is instructive to review these methods briefly, to provide some context for our NBO approach. These methods either directly approximate the $Q$-value $Q(b, a)$ or indirectly approximate the $Q$-value by approximating the cost-to-go $J^{*}(b)$, and include heuristic expected ECTG [28], parametric approximation [29, 30], policy rollout [31], hindsight optimization [32,33], and foresight optimization (also called open-loop feedback control (OLFC)) [25]. The following is a summary of these methods, exposing the nature of each approximation (for a detailed discussion of these methods applied to sensor resource management problems, see [15]):

(i) heuristic ECTG:

$$
Q(b, a) \approx c(b, a)+\gamma N(b, a)
$$

(ii) parametric approximation (e.g., Q-learning):

$$
Q(b, a) \approx \hat{Q}(b, a, \theta),
$$

(iii) policy rollout:

$$
Q(b, a) \approx c(b, a)+\mathrm{E}\left[J^{\pi_{\text {base }}}\left(b^{\prime}\right) \mid b\right],
$$

(iv) hindsight optimization:

$$
J^{*}(b) \approx \mathrm{E}\left[\min _{\left(a_{k}\right)_{k}} \sum_{k} c\left(b_{k}, a_{k}\right) \mid b\right]
$$

(v) foresight optimization (OLFC):

$$
J^{*}(b) \approx \min _{\left(a_{k}\right)_{k}}\left[\sum_{k} c\left(b_{k}, a_{k}\right) \mid b,\left(a_{k}\right)_{k}\right] .
$$

The notation $\left(a_{k}\right)_{k}$ means the ordered list $\left(a_{0}, a_{1}, \ldots\right)$. Typically, the expectations in the last three methods are approximated using Monte Carlo methods.

The NBO approach may be summarized as follows:

$$
J^{*}(b) \approx \min _{\left(a_{k}\right)_{k}} \sum_{k} c\left(\hat{b}_{k}, a_{k}\right),
$$

where $\left(\hat{b}_{k}\right)_{k}$ represents a nominal sequence of belief states. Thus, it resembles both the hindsight and foresight optimization approaches, but with the expectation approximated by one sample. The reader will notice that hindsight and foresight optimizations differ in the order in which the expectation and minimization is taken. However, because NBO involves only a single sample path (instead of an expectation), NBO straddles this distinction between hindsight and foresight optimization.

The central motivation behind $\mathrm{NBO}$ is computational efficiency. If one cannot afford to simulate multiple samples of the random noise sequences to estimate expectations, and only one realization can be chosen, it is natural to choose the "nominal" sequence (e.g., maximum likelihood or mean). The nominal noise sequence leads to a nominal belief-state sequence $\left(\hat{b}_{k}\right)_{k}$ as a function of the chosen action sequence $\left(a_{k}\right)_{k}$. Note that in NBO, as in foresight optimization, the 
optimization is over a fixed sequence $\left(a_{k}\right)_{k}$ rather than a noise-dependent sequence or a policy.

There are two points worth emphasizing about the NBO approach. First, the nominal belief-state sequence is not fixed, as (27) might suggest; rather, the underlying random variables are fixed at nominal values and the belief states become deterministic functions of the chosen actions. Second, the expectation implicit in the incremental cost $c\left(\hat{b}_{k}, a_{k}\right)$ (recall (7) and (12)) need not be approximated by the "nominal" value. In fact, for the mean-squared-error cost we use in the tracking application, the nominal value would be 0 . Instead, we use the fact that the expected cost can be evaluated analytically by (21) under the previously stated assumptions of correct tracking model, Gaussian statistics, and correct data association.

Because NBO approximates the belief-state evolution but not the cost evaluation, the method is suitable when the primary effect of the randomness appears in the cost, not in the state prediction. Thus, NBO should perform well in our tracking application as long as the target motion is reasonably predictable with the tracking model within the chosen planning horizon.

The general procedure for using the $\mathrm{NBO}$ approximation may be summarized as follows.

(1) Write the state dynamics as functions of zero-mean noise. For example, borrowing from the notation of Section 3.2:

$$
\begin{aligned}
x_{k+1} & =f\left(x_{k}, a_{k}\right)+v_{k}, \quad v_{k} \sim \mathcal{N}\left(0, Q_{k}\right), \\
z_{k} & =g\left(x_{k}\right)+w_{k}, \quad w_{k} \sim \mathcal{N}\left(0, R_{k}\right) .
\end{aligned}
$$

(2) Define nominal belief-state sequence $\left(\hat{b}_{1}, \ldots, \hat{b}_{H-1}\right)$

$$
\begin{gathered}
b_{k+1}=\Phi\left(b_{k}, a_{k}, v_{k}, w_{k+1}\right) \Longrightarrow \hat{b}_{k+1}=\Phi\left(\hat{b}_{k}, a_{k}, 0,0\right), \\
\hat{b}_{0}=b_{0},
\end{gathered}
$$

in the linear Gaussian case, this is the MAP estimate of $b_{k}$.

(3) Replace expectation over random future belief states

$$
J_{H}\left(b_{0}\right)=\mathrm{E}_{b_{1}, \ldots, b_{H}}\left[\sum_{k=1}^{H} c\left(b_{k}, a_{k}\right)\right]
$$

with the sample given by nominal belief state sequence

$$
J_{H}\left(b_{0}\right) \approx \sum_{k=1}^{H} c\left(\hat{b}_{k}, a_{k}\right) .
$$

(4) Optimize over action sequence $\left(a_{0}, \ldots, a_{H-1}\right)$.

As pointed out before, because our focus here is to introduce $\mathrm{NBO}$ as a new approximation method, the optimization in the last step above is taken to be a generic optimization problem that is solved using a generic method. In our simulation studies, we used Matlab's fmincon function.
In the specific case of tracking, recall that the belief state $b_{k}^{\zeta}$ corresponding to the target state $\zeta_{k}$ is identified with the track state $\left(\xi_{k}, P_{k}\right)$ according to (20). Therefore, the nominal belief state $\hat{b}_{k}^{\zeta}$ evolves according to the nominal track state trajectory $\left(\hat{\xi}_{k}, \hat{P}_{k}\right)$ given by the (extended) Kalman filter equations with an exactly zero noise sequence. This reduces to the following:

$$
\begin{aligned}
\hat{b}_{k}^{\zeta}(\zeta) & =\mathcal{N}\left(\zeta-\hat{\xi}_{k}, \hat{P}_{k}\right), \\
\hat{\xi}_{k+1} & =F_{k} \hat{\xi}_{k}, \\
\hat{P}_{k+1} & =\left[\left(F_{k} \hat{P}_{k} F_{k}^{T}+Q_{k}\right)^{-1}+H_{k+1}^{T}\left[R_{k+1}\left(\hat{\xi}_{k}, s_{k}\right)\right]^{-1} H_{k+1}\right]^{-1},
\end{aligned}
$$

where the (linearized) target motion model is given by the following:

$$
\begin{aligned}
\zeta_{k+1} & =F_{k} \zeta_{k}+v_{k}, \quad v_{k} \sim \mathcal{N}\left(0, Q_{k}\right), \\
z_{k} & =H_{k} \zeta_{k}+w_{k}, \quad w_{k} \sim \mathcal{N}\left(0, R_{k}\left(\zeta_{k}, s_{k}\right)\right) .
\end{aligned}
$$

The incremental cost given by the nominal belief state is then

$$
c\left(\hat{b}_{k}, a_{k}\right)=\operatorname{Tr} \hat{P}_{k+1}=\sum_{i=1}^{N_{\text {targ }}} \operatorname{Tr} \hat{P}_{k+1}^{i},
$$

where $N_{\text {targ }}$ is the number of targets.

4.2. Finite Horizon. In the guidance problem we are interested in long-term tracking performance. For the sake of exposition, if we idealize this problem as an infinite-horizon POMDP (ignoring the attendant technical complications), Bellman's principle can be stated as follows:

$$
J_{\infty}^{*}\left(b_{0}\right)=\min _{\pi} \mathrm{E}\left[\sum_{k=0}^{H-1} c\left(b_{k}, \pi\left(b_{k}\right)\right)+J_{\infty}^{*}\left(b_{H}\right)\right]
$$

for any $H<\infty$. The term $\mathrm{E}\left[J_{\infty}^{*}\left(b_{H}\right)\right]$ is the ECTG from the end of the horizon $H$. If $H$ represents the practical limit of horizon length, then (35) may be approximated in two ways:

$$
\begin{aligned}
& J_{\infty}^{*}\left(b_{0}\right) \approx \min _{\pi} \mathrm{E}\left[\sum_{k=0}^{H-1} c\left(b_{k}, \pi\left(b_{k}\right)\right)\right] \text { (truncation), } \\
& J_{\infty}^{*}\left(b_{0}\right) \approx \min _{\pi} \mathrm{E}\left[\sum_{k=0}^{H-1} c\left(b_{k}, \pi\left(b_{k}\right)\right)+\hat{J}\left(b_{H}\right)\right] \text { (HECTG). }
\end{aligned}
$$

The first amounts to ignoring the ECTG term, and is often the approach taken in the literature. The second replaces the exact ECTG with a heuristic approximation, typically a gross approximation that is quick to compute. To benefit from the inclusion of a heuristic ECTG (HECTG) term in the cost function for optimization, $\widehat{J}$ needs only to be a better estimate of $J_{\infty}^{*}$ than a constant. Moreover, the utility of the approximation is in how well it rank actions, not in how well it estimates the ECTG. Section 5.4 will illustrate the crucial role this term can play in generating a good action policy. 


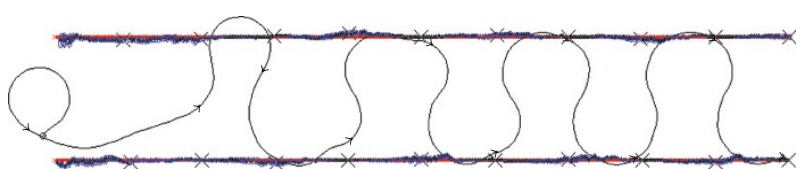

Figure 1: No occlusion with $H=1$.

\section{Single UAV Case}

We begin our assessment of the performance of a POMDPbased design with the simple case of a single UAV and two targets, where the two targets move along parallel straightline paths. This is enough to demonstrate the qualitative behavior of the method. It turns out that a straightforward but naive implementation of the POMDP approach leads to performance problems, but these can be overcome by employing an approximate ECTG term in the objective, and a two-phase approach for the action search.

5.1. Scenario Trajectory Plots. First, we describe what is depicted in the scenario trajectory plots that appear throughout the remaining sections. See, for example, Figures 1 and 2. Target location at each measurement time is indicated by a small red dot. The targets in most scenarios move in straight horizontal lines from left to right at constant speed. The track covariances are indicated by blue ellipses at each measurement time; these are 1-sigma ellipses corresponding to the position component of the covariances, centered at the mean track position indicated by a black dot. (However, this coloring scheme is modified in later sections in order to better distinguish between closely spaced targets.)

The UAV trajectory is plotted as a thin black line, with an arrow periodically. Large X's appear on the tracks that are synchronized with the arrows on the UAV trajectory, to give a sense of relative positions at any time.

Finally, occlusions are indicated by thick light green lines. When the line of sight from a sensor to a target intersects an occlusion, that target is not visible from that sensor. This is a crude model of buildings or walls that block the visibility of certain areas of the ground from different perspectives. It is not meant to be realistic, but serves to illustrate the effect of occlusions on the performance of the UAV guidance algorithm.

5.2. Results with No ECTG. Following the NBO procedure, our first design for guiding the UAV optimizes the cost function (31) within a receding horizon approach, issuing only the command $a_{0}$ and reoptimizing at the next step. In the simplest case, the policy is a myopic one: choose the next action that minimizes the immediate cost at the next step based on current state information. This is equivalent to a receding horizon approach with $H=1$ and no ECTG term. The behavior of this policy in a scenario with two targets moving at constant velocity along parallel paths is illustrated in Figure 1. For this scenario, the behavior with $H>1$ (applying NBO) is not qualitatively different. The UAV's speed is greater than the targets', so the UAV is forced to loop or weave to reduce its average speed. Moreover, the

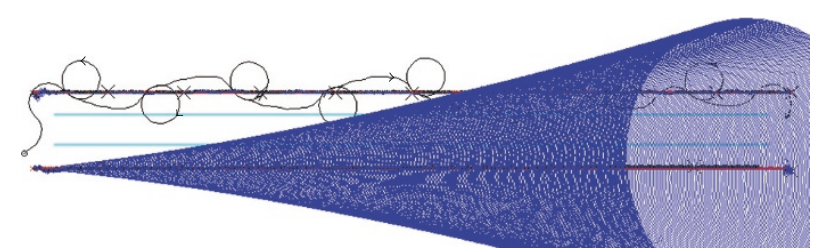

FIgURE 2: Gap occlusion with $H=1$.

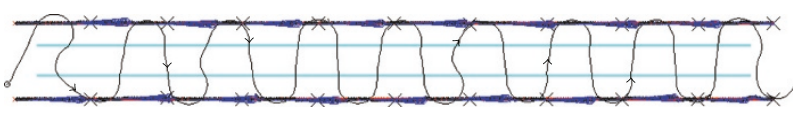

Figure 3: Gap occlusion with $H=4$.

UAV tends to fly over one target than the other, instead of staying in between. There are two main reasons for this. First, the measurement noise is nonisotropic, so it is beneficial to observe the targets from different angles over time. Second, the trace objective is minimized by locating the UAV over the target with the greater covariance trace.

To see this, consider a simplified one-dimensional tracking problem with stationary targets on the real line with positions $x_{1}$ and $x_{2}$, sensor position $y$, and noisy measurement of target positions given by

$$
z_{i} \sim \mathcal{N}\left(x_{i}, \rho\left(y-x_{i}\right)^{2}+r\right), \quad i=1,2 .
$$

This noise model is analogous to the relative range uncertainty defined in Section 3.2. If the current "track" variances are given by $p_{1}$ and $p_{2}$, then the variances after updating with the Kalman filter, as a function of the new sensor location $y$, are given by

$$
p_{i}^{+}(y)=\left(1-k_{i}\right) p_{i}=\frac{\rho\left(y-x_{i}\right)^{2}+r}{\rho\left(y-x_{i}\right)^{2}+r+p_{i}} p_{i}, \quad i=1,2,
$$

and the trace of the overall (diagonal) covariance is $c(y)=$ $p_{1}^{+}(y)+p_{2}^{+}(y)$. It is not hard to show that if the targets are separated enough, $c(y)$ has local minima at about $y=x_{1}$ and $y=x_{2}$ with values of approximately $p_{2}+p_{1} r /\left(p_{1}+r\right)$ and $p_{1}+p_{2} r /\left(p_{2}+r\right)$, respectively. Therefore, the best location of the sensor is at about $x_{1}$ if $p_{1}>p_{2}$, and at about $x_{2}$ if the opposite is true.

Thus, the simple myopic policy behaves in a nearly optimal manner when there are no occlusions. However, if occlusions are introduced, some lookahead (e.g., longer planning horizon) is necessary to anticipate the loss of observations. Figure 2 illustrates what happens when the planning horizon is too short. In this scenario, there are two horizontal walls with a gap separating them. If the UAV cannot cross the gap within the planning horizon, there is no apparent benefit to moving away from the top target toward the bottom target, and the track on the bottom target goes stale. On the other hand, with $H=4$ the horizon is long enough to realize the benefit of crossing the gap, and the weaving behavior is recovered (see Figure 3 ). 


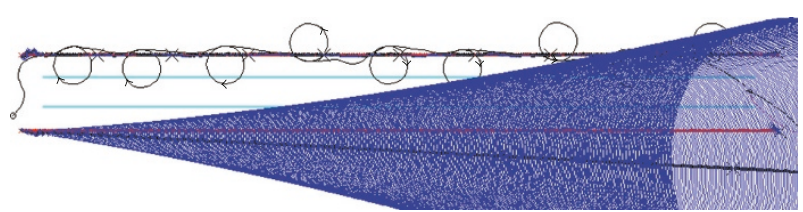

FIGURE 4: Gap occlusion with $H=4$, search initialized with $H=1$ plan.

In addition, to the length of the planning horizon, another factor that can be important in practical performance is the initialization of the search for the action sequence. The result of the policy of initializing the fourstep action sequence with the output of the myopic plan $(H=1)$ is shown in Figure 4. The search fails to overcome the poor performance of the myopic plan because the search starts near a local minimum (recall that the trace objective has local minima in the neighborhood of each target). Bellman's principle depends on finding the global minimum, but our search is conducted with a gradient-based algorithm (Matlab's fmincon function), which is susceptible to local minima. One remedy is to use a more reliable but expensive global optimization algorithm. Another remedy, the one we chose, is to use a more intelligent initialization for the search, using a penalty term described in the next section.

5.3. Weighted Trace Penalty. The performance failures illustrated in the previous section are due to the lack of sensitivity in our finite-horizon objective function (31) to the cost of not observing a target. When the horizon is too short, it seems futile to move toward an unobserved target if no observations can be made within the horizon. Likewise, if the action plan required to make an observation on an occluded target deviates far enough from the initial plan, it may not be found by a local search because locally there is no benefit to moving toward the occluded target. To produce a solution closer to the optimal infinite-horizon policy, the benefit of initial actions that move the UAV closer to occluded targets must be exposed somehow.

One way to expose that benefit is to augment the cost function with a term that explicitly rewards actions that bring the UAV closer to observing an occluded target. However, such modifications must be used with caution. The danger of simply optimizing a heuristically modified cost function is that the heuristics may not apply well in all situations. Bellman's principle informs us of the proper mechanism to include a term modeling a "hidden" long-term cost: the ECTG term. Indeed, the blame for poor performance may be placed on the use of truncation rather than HECTG as the finite-horizon approximation to the infinite-horizon cost (see Section 4.2).

In our tracking application, the hidden cost is the growth of the covariance of the track on an occluded target while it remains occluded. We estimate this growth by a weighted trace penalty (WTP) term, which is a product of the current covariance trace and the minimum distance to observability (MDO) for a currently occluded target, a term we define precisely below. With the UAV moving at a constant speed,

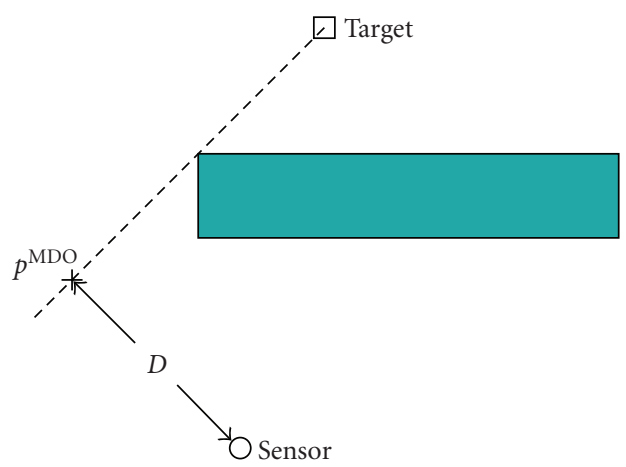

Figure 5: Minimum distance to observability.

this is roughly equivalent to a scaling of the trace by the time it takes to observe the target. When combined with the trace term that is already in the cost function, this amounts to an approximation of the track covariance at the time the target is finally observed. More accurate approximations are certainly possible, but this simple approximation is sufficient to achieve the desired effect.

Specifically, the terminal cost or ECTG term using the WTP has the following form:

$$
\widehat{J}(b)=J_{\mathrm{WTP}}(b):=\gamma D\left(s, \xi^{\mathbf{i}}\right) \operatorname{Tr} P^{\mathbf{i}},
$$

where $\gamma$ is a positive constant, $\mathbf{i}$ is the index of the worst occluded target

$$
\begin{aligned}
& \mathbf{i}=\underset{i \in \ell}{\operatorname{argmax}} \operatorname{Tr} P^{i}, \\
& \iota=\left\{i \mid \xi^{i} \text { invisible from } s\right\},
\end{aligned}
$$

and $D(s, \xi)$ is the MDO, that is, the distance from the sensor location given by $s$ to the closest point $p^{\mathrm{MDO}}(s, \xi)$ from which the target location given by $\xi$ is observable. Figure 5 is a simple illustration of the MDO concept. Given a single rectangular occlusion, $p^{\mathrm{MDO}}(s, \xi)$ and $D(s, \xi)$ can be found very easily. Given multiple rectangular occlusions, the exact MDO is cumbersome to compute, so we use a fast approximation instead. For each rectangular occlusion $j$, we compute $p_{j}^{\mathrm{MDO}}(s, \xi)$ and $D_{j}(s, \xi)$ as if $j$ were the only occlusion. Then we have $D(s, \xi) \geq \max _{j} D_{j}(s, \xi)>0$ whenever $\xi$ is occluded from $s$, so we use $\max _{j} D_{j}(s, \xi)$ as a generally suitable approximation to $D(s, \xi)$.

The reason a worst-case among the occluded targets is selected, rather than including a term for each occluded target, is that this forces the UAV to at least obtain an observation on one target instead of being pulled toward two separate targets and possibly never observing either one. The true ECTG certainly includes costs for all occluded targets. However, given that the ECTG can only be approximated, the quality of the approximation is ultimately judged by whether it leads to the correct ranking of action plans within the horizon, and not by whether it closely models the true ECTG value. We claim that by applying the penalty to only the worst track covariance, the chosen actions are closer to the optimal policy than what would result by applying the penalty to all occluded tracks. 


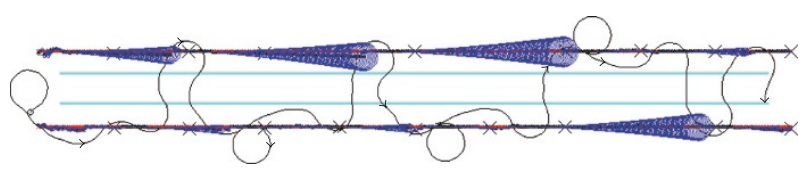

FIgURE 6: Behavior of WTP(1).

5.4. Results with WTP for ECTG. Let WTP $(H)$ denote the procedure of optimizing the NBO cost function with horizon length $H$ plus the WTP estimate of the ECTG:

$$
\min _{a_{0}, \ldots, a_{H-1}} \sum_{k=0}^{H-1} c\left(\hat{b}_{k}, a_{k}\right)+J_{\mathrm{WTP}}\left(\hat{b}_{H}\right)
$$

Initially, we consider the use of $\mathrm{WTP}(1)$ in two different roles: adapting the horizon length and initializing the action search. Subsequently, we consider the effect of the terminal cost in $\operatorname{WTP}(H)$ with $H>1$.

Figure 6 shows the behavior of $\mathrm{WTP}(1)$ on the gap scenario previously considered, using a penalty weight of just $\gamma=10^{-6}$. Comparing with Figure 2, which has the same horizon length but no penalty term, we see that the WTP has the desired effect of forcing the UAV to alternately visit each target. Therefore, the output of $\mathrm{WTP}(1)$ is a reasonable starting point for predicting the trajectory arising from a good action plan. Since WTP(1) is really a form of Q-value approximation (namely, the heuristic ECTG approach mentioned in the beginning of Section 4.1), it is not surprising that it generates a nonmyopic policy that outperforms the myopic policy, even though both policies evaluate the incremental cost $c$ at only one step.

By playing out a sequence of applications of WTP (1) — which amounts to a sequence of one-dimensional optimizations-we can quickly generate a prediction of sensor motion that is useful for adapting the planning horizon and initializing the multistep action search, potentially mitigating the effects seen in Figures 2 and 4 . Thus, we use a three-step algorithm described as follows.

(1) Generate an initial action plan by a sequence of $H_{\max }$ applications of WTP(1).

(2) Choose $H$ to be the minimum number of steps such that there is no change in observability of any of the targets after that time, with a minimum value of $H_{\min }$.

(3) Search for the optimal $H$-step action sequence, starting at the initial plan generated in step 1.

This can be considered a two-phase approach, with the first two steps constituting Phase I and the third step being Phase II. The heuristic role of $\mathrm{WTP}(1)$ in the above algorithm is appropriate in the POMDP framework, because any suboptimal behavior caused by the heuristic in Phase I has a chance of being corrected by the optimization over the longer horizon in Phase II, provided $H_{\min }$ and $H_{\max }$ are large enough. Figure 7 shows the effectiveness of using WTP $(1)$ to choose $H$ and initialize the search. In this test, $H_{\min }=1$ and $H_{\max }=8$, and the mean value of the adaptive $H$ is 3.7, which

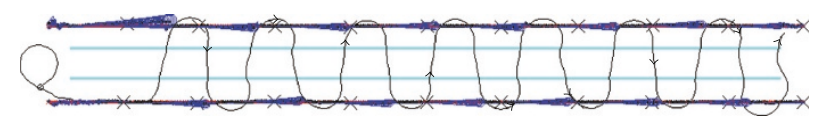

FIgURE 7: WTP(1) used for initialization and adaptive horizon.

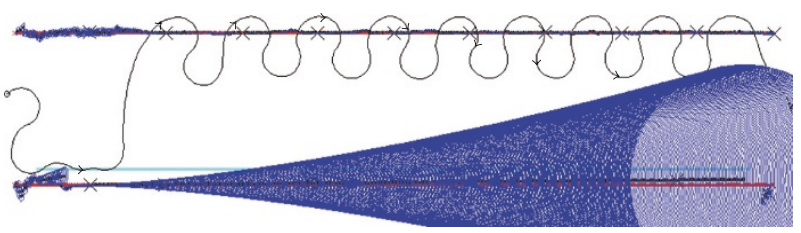

FIGURE 8: Effect of truncated horizon with no ECTG.

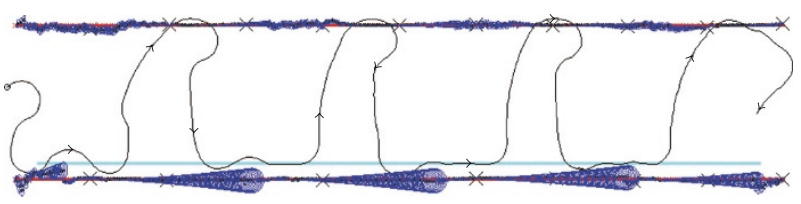

Figure 9: Behavior of WTP $(H)$ policy.

corresponds approximately to $H=4$ in Figure 3 but without having to identify that value beforehand.

In practice, however, the horizon length is always bounded above in order to limit the computation in any planning iteration, and the upper bound $H_{\max }$ may sometimes be too small to achieve the desired performance. Figure 8 illustrates such a scenario. There is only one occlusion, but it is far enough from the upper target that once the UAV moves sufficiently far from the occlusion, the horizon is too short to realize the benefit of heading toward the lower target when minimizing the trace objective. This is despite the fact that the search is initialized with the UAV headed straight down according to $\mathrm{WTP}(1)$.

The remedy, of course, is to use WTP as the ECTG in Phase II, that is, to employ $\operatorname{WTP}(H)$ as in (41). The effect of $\operatorname{WTP}(H)$ is depicted in Figure 9. In general, the inclusion of the ECTG term makes lookahead more robust to poor initialization and short horizons.

In general, we would not expect the optimal trajectory to be symmetric with respect to the two targets, because of a number of possible factors, including: (1) the location of the occlusions, and (2) the dynamics and the acceleration constraints on the UAV. In Figures 6 and 9, we see this asymmetry in that the UAV does not spend equal amounts of time near the two targets. In Figure 9, the position of the occlusion is highly asymmetric in relation to the path of the two targets-in this case, it is not surprising that the UAV trajectory is also asymmetric. In Figure 6, the two occlusions are more symmetric, and we would expect a more symmetric trajectory in the long run. However, in the short run, the UAV trajectory is not exactly symmetric because of the timing and direction of the UAV as it crosses the occlusion. The particular timing and direction of the UAV results in the need for an extra loop in some instances but not others. 


\section{Multiple UAV Case}

As it stands, the procedure developed for the single UAV case is ill-suited to the case of multiple UAVs, because the WTP is defined with only a single sensor in mind. An extension of the WTP to multiple sensors is developed in Section 6.1, and in Section 6.2 this extension is applied to a new scenario to demonstrate the coordination of two sensors.

6.1. Extension of WTP. A slight modification of the WTP defined in (39) can certainly be used as an ECTG in scenarios with more than one sensor, for example:

$$
\widehat{J}(b)=\gamma \min _{j} D\left(s^{j}, \xi^{\mathbf{i}}\right) \operatorname{Tr} P^{\mathbf{i}},
$$

where $s^{j}$ is the state of sensor $j$. However, this underutilizes the sensors, because only one sensor can affect the ECTG. One would like the ECTG to guide two sensors toward two separate occluded targets if it makes sense to do so. On the other hand, if one sensor can "cover" two occluded targets efficiently, there is no need to modify the motion of a second sensor. The problem, therefore, is to decide which sensor will receive responsibility for each occluded target.

It is natural to assign the "nearest" sensor to an occluded target, that is, the one that minimizes the MDO as in (42). However, to account for the effect of previous assignments to that sensor, the MDO should not be measured along a straight line directly from the starting position of the sensor, but rather, along the path the sensor takes while making observations on previously assigned targets. In the spirit of the WTP for a single sensor, it is assumed that if multiple occluded targets are assigned to a sensor, the most uncertain track (the one with the highest covariance trace) is the one that appears in the WTP and governs the motion of the sensor, until the target is actually observed; then, the next most uncertain track appears in the WTP, and so on. So, roughly speaking, the sensor makes observations of occluded targets in order of decreasing uncertainty.

Therefore, a multiple weighted trace penalty (MWTP) term is computed according to the following procedure.

(1) Find the set of targets occluded from all sensors, and sort in order of decreasing $\operatorname{Tr} P^{i}$.

(2) Set $\hat{J}=0$, and $D_{j}=0$ for each sensor $j$.

(3) For each occluded target $i$ (in order):
(a) find $\mathbf{j}=\operatorname{argmin}_{j}\left\{D_{j}+D\left(s^{j}, \xi^{i}\right)\right\}$;
(b) if $D_{\mathbf{j}}=0$ then set $\hat{J} \leftarrow \hat{J}+\gamma D\left(s^{\mathbf{j}}, \xi^{i}\right) \operatorname{Tr} P^{i}$;
(c) set $D_{\mathbf{j}} \leftarrow D_{\mathbf{j}}+D\left(s^{\mathbf{j}}, \xi^{i}\right)$ and $s^{\mathbf{j}} \leftarrow p^{\mathrm{MDO}}\left(s^{\mathbf{j}}, \xi^{i}\right)$.

This procedure is an approximation in several respects. First, it ignores the motion of the targets in the interval of time it takes the sensor to move from one $p^{\mathrm{MDO}}$ location to the next. Second, it ignores the dynamic constraints of the UAVs. The total distance is computed by a greedy, suboptimal algorithm. None of these deficiencies is insurmountable, but for the purpose of a quick heuristic ECTG for ranking action plans, this MWTP is sufficient.

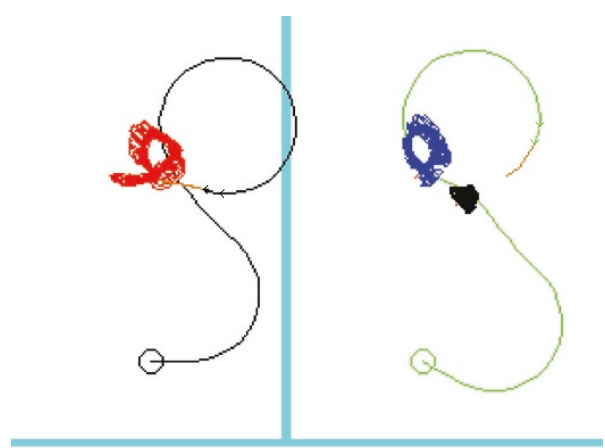

FIGURE 10: Beginning of scenario: sensors cover separate regions.

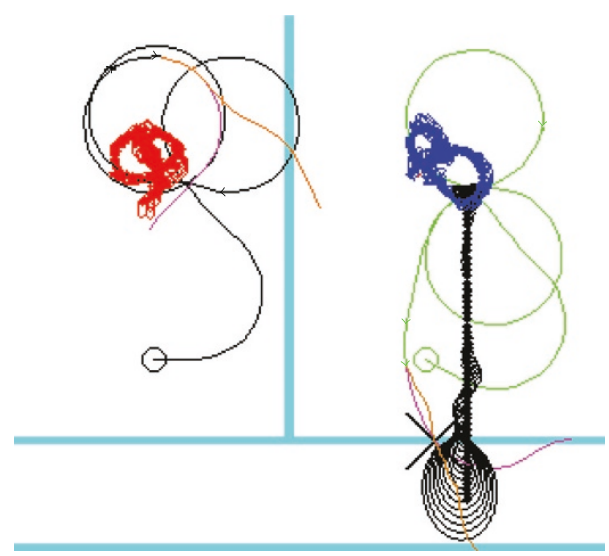

FIGURE 11: Transition: sensors coordinate plans to cover all targets as one target moves.

6.2. Coordinated Sensor Motion. Figures 10, 11, and 12 show snapshots of a scenario illustrating the coordination capability of the guidance algorithm using the MWTP from the previous section as an ECTG term. There are three targets (red, blue, and black) and two sensor UAVs (black and green). This scenario also demonstrates the adaptive horizon, with thin magenta and orange lines showing the UAVs' planned Phase I and Phase II trajectories, respectively, according to the current horizon length $H$.

Initially, the three targets are divided into two regions by an occlusion, and one sensor covers each region. At this point $H=1$ is a sufficient horizon. Then, the black target heads down and crosses two occlusions to enter the bottom region. In response, the green UAV chases after the downwardbound target, while the black UAV moves to cover both upper regions - the sensors coordinate to maximize coverage of the targets. Figure 11 plots the UAV motion plans at the moment the planner decides to chase the downward-bound target. A large black X marks the spot from which the green sensor expects to first see the black target. Generally speaking, the longer the planning horizon, the earlier the UAVs react to the downward-bound target, and the less time any target remains unseen by a sensor. In the moment depicted in the figure, 


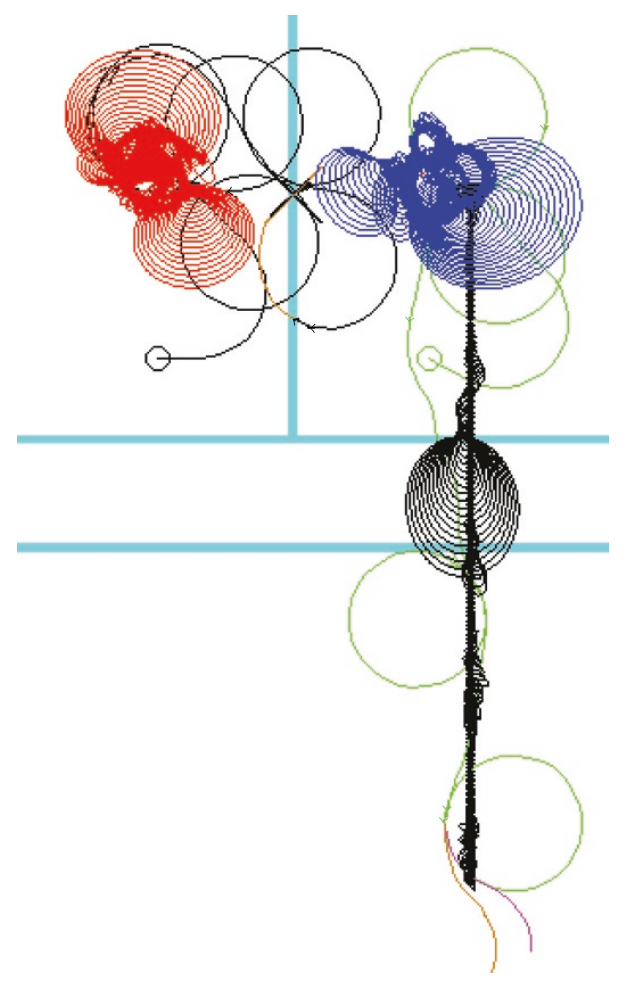

FIGURE 12: End of scenario: sensors have coordinated for maximum coverage.

Phase I has predicted that the black target is going to cross the occlusion, and thus the adaptive horizon has increased to $H=6$.

Unlike the previous scenarios, this scenario features random target motion as well as random measurement noise. This allows a broader comparison of performance among different planning algorithms. Figure 13 shows a plot of the empirical cumulative distribution function (CDF) of the average tracking performance of seven algorithms: $H=$ 1 with no ECTG term, $\operatorname{MWTP}(1), \operatorname{MWTP}(3), \operatorname{MWTP}(4)$, $\operatorname{MWTP}(5), \operatorname{MWTP}(6)$, and $\operatorname{MWTP}(H)$ with adaptive $H$ between 1 and 6 . The plot shows that the use of the approximate ECTG produces substantially better performance. Without the MWTP term in the objective, one of the targets (usually the downward-bound one) is ignored when it becomes occluded. There appears to be a minor benefit to using $H=1$ or adaptive horizon over the other settings. However, one should not make too much of this apparent ranking. Perturbations of the problem configuration or other parameters result in other performance rankings, though in all cases MWTP significantly outperforms the pure myopic policy lacking ECTG.

\section{Track Ambiguity}

Track accuracy metrics, such as the mean-squared-error metric proposed in Section 3.2, are not the only measure of tracking performance. Other considerations such as track

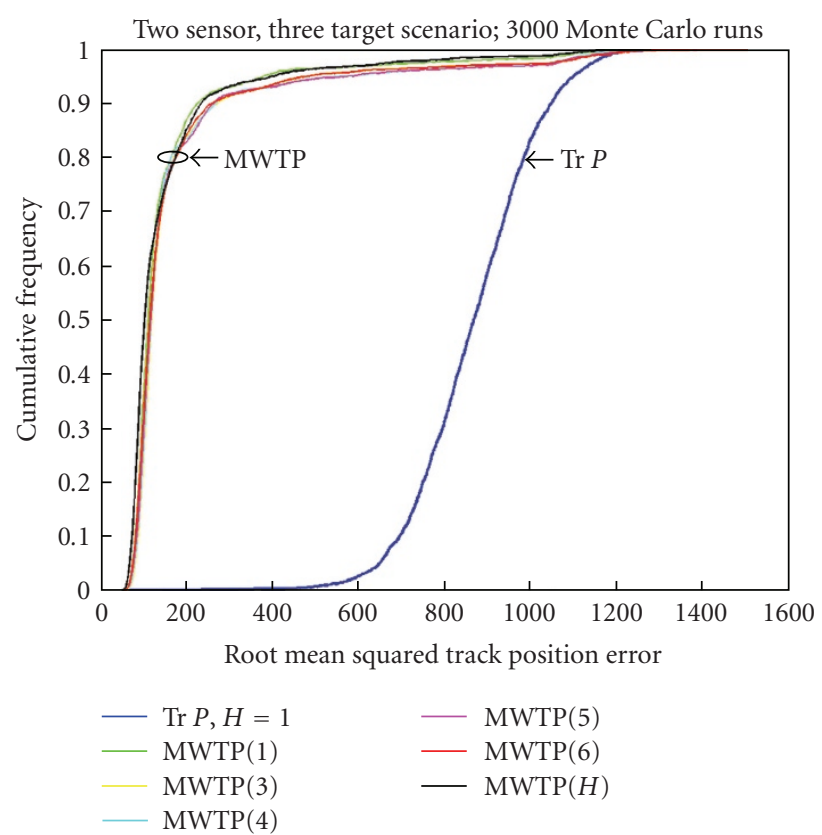

FIGURE 13: CDF of tracking performance in multi-sensor scenario.

duration and track continuity are also important. In particular, when target ID or threat class information is attached to a track through some separate discrimination process, it is important to maintain a consistent association between the track and the target it represents. So-called "track swaps" (switches in the mapping between targets and tracks) may be caused by incorrect data association-updating a track with measurements from a different target-or by approximation of the true Bayesian update of the target state distribution that the track state represents. The latter cause is mainly a function of the tracking algorithm; the multiple hypothesis tracking (MHT) algorithm with an unlimited hypothesis set represents the true Bayesian update under standard assumptions [34], but any practical tracker is an approximation of the ideal. Data association ambiguity, on the other hand, is a function of the sensor locations as well as the tracker, and therefore minimizing this quantity is a suitable objective in the UAV guidance problem. In this section, we demonstrate the flexibility of the POMDP framework by augmenting the mean-squared-error cost function with a term that represents the risk of a track swap, and applying the same basic algorithm to demonstrate how the guidance algorithm reduces the probability of a track swap in a scenario where the targets are confusable.

7.1. Detecting Ambiguity. A challenge of this exercise is that it is hard to predict track swaps with $\mathrm{NBO}$, since the full spectrum of uncertainty is not explored. In the context of predicting the performance of a proposed action sequence, one could try to detect a track swap by comparing associations of predicted track states and predicted target states. This approach might work within a Monte Carlo approximation method such as hindsight optimization, foresight optimization, or policy rollout. With NBO, however, 
the only predicted target state is the one that comes from the maximum likelihood value of the predicted track state, so the best data association will always be the "correct" one. We must resort to a more indirect approach, measuring a quantity that serves as a predictor of a likely track swap.

The assessment of data association ambiguity is currently a topic of concern in tracking [35], because of its role as an indicator of the potential for error in track states and track identity. Nevertheless, the ambiguity of a single measurement-to-track data association is not a reliable predictor of track swap. Consider the case of two targets that cross each other at an oblique angle, which are tracked with an NCV model updated with position measurements. Despite the complete ambiguity of association at the point when the targets cross, a track swap is extremely unlikely under a reasonable track update rate because the velocity estimate is unaffected by the ambiguity. Furthermore, tracks can become confusable after accumulating a series of updates with slightly ambiguous data associations, none of which is egregious enough by itself to indicate trouble. This suggests using an extended period of data association ambiguity as a predictor of track swap; however, one can easily envision a scenario in which one or two misassociations is enough to cause a track swap.

Similarity of target state distributions (belief states) should be a better indicator of the potential for a track swap. If two tracks have similar distributions, it is unlikely that the targets they represent can be reliably discriminated from each other, now or in the future. For this approach to work, the belief-state updates must reflect the inherent ambiguity of the target states. It will not suffice to use a single-hypothesis tracker in the prediction of belief states, even if the data association is correct as it is in the "truth tracker" used elsewhere in this paper. Again, a full MHT algorithm is required to represent the true Bayesian update of the belief states, which is intractable exactly when data association is ambiguous. We have found that when the hypothesis set is truncated to a reasonable limit, the MHT has trouble representing uncertainty over extended periods of time. Instead, we use the joint probability data association (JPDA) algorithm [36] for belief-state (and track-state) updates in this context, because it is designed to represent track state uncertainty but in the compressed representation of one Gaussian distribution per track.

The dissimilarity between distributions may be measured in several ways: Kullback-Leibler divergence (or alpha divergence), Bhattacharyya distance, or discordance [37], all of which have closed-form solutions for Gaussians. However, these measures are basically average-case measures of how often the state values from the two distributions are within a small neighborhood of each other. It turns out that a worst-case metric is a better predictor of the potential for a track swap. The reason for this is that track swaps are more closely associated with instantaneous ambiguities in the track associations. Specifically, even if on average the state variables from two tracks are not often close, even a single occurrence of an ambiguous measurement can cause a track swap. The worst-case metric we use is defined next.
Given a Gaussian distribution $\mathcal{N}(\mu, P)$, define the " $\chi^{2}$ value" as follows:

$$
\chi_{\mu, P}^{2}(x):=(x-\mu)^{T} P^{-1}(x-\mu),
$$

so called because when $x \sim \mathcal{N}(\mu, P)$ the quantity has an $\chi^{2}$ distribution with $n$ degrees of freedom, where $n$ is the number of components in $x$. This is the square of the Mahalanobis distance from $\mu$ to $x$. We define a worst-case " $\chi 2$ distance" between two Gaussian distributions $\mathcal{N}\left(\mu_{1}, P_{1}\right)$ and $\mathcal{N}\left(\mu_{2}, P_{2}\right)$ as follows:

$$
\begin{aligned}
D_{\chi^{2}}\left(\mu_{1}, P_{1} ; \mu_{2}, P_{2}\right) & \\
& :=\min \left\{d \mid \exists x \ni \chi_{\mu_{1}, P_{1}}^{2}(x) \leq d, \chi_{\mu_{2}, P_{2}}^{2}(x) \leq d\right\} \\
& =\min _{x} \max \left\{\chi_{\mu_{1}, P_{1}}^{2}(x), \chi_{\mu_{2}, P_{2}}^{2}(x)\right\} .
\end{aligned}
$$

Note that it makes sense to compare $\chi_{\mu_{1}, P_{1}}^{2}(x)$ and $\chi_{\mu_{2}, P_{2}}^{2}(x)$ since they have the same distribution when $x$ is drawn randomly from $\mathcal{N}\left(\mu_{1}, P_{1}\right)$ and $\mathcal{N}\left(\mu_{2}, P_{2}\right)$, respectively. Geometrically, $D_{\chi^{2}}$ may be interpreted as the smallest $d$ such that the ellipsoid level surfaces $\chi_{\mu_{1}, P_{1}}^{2}(x)=d$ and $\chi_{\mu_{2}, P_{2}}^{2}(x)=$ $d$ just touch each other. Analytically, the problem may be seen as measuring the distance between $\mu_{1}$ and $\mu_{2}$ but using two different distance metrics. One way to use two different metrics is to consider the set of points "equidistant" from the two means, that is, the points having the same distance from each mean using the applicable Mahalanobis distance from each mean. Then, the desired distance is given by the equidistant point with the least distance. Strictly speaking, $D_{\chi^{2}}$ (or its square root) is not a distance because it does not satisfy the triangle inequality, but it does satisfy symmetry and positivity, with a value of zero only when the means agree.

The computation of $D_{\chi^{2}}$ is a quasiconvex problem, which can be solved with a bisection method involving a generalized eigenvalue problem at each iteration, according to the Sprocedure [38]. This is a rather expensive procedure to execute as part of a single objective function evaluation. However, empirical tests revealed that one of the upper bounds used in the bisection method tends to be a constant factor of the true value in both ambiguous and unambiguous situations, so we elected to use that as a surrogate for $D_{\chi^{2}}$. The upper bound in question is obtained by restricting the problem to the line segment between $\mu_{1}$ and $\mu_{2}$ :

$$
\begin{array}{r}
\hat{D}_{\chi^{2}}\left(\mu_{1}, P_{1} ; \mu_{2}, P_{2}\right):=\min _{\alpha \in[0,1]} \max \left\{\chi_{\mu_{1}, P_{1}}^{2}\left(\mu_{1}+\alpha\left(\mu_{2}-\mu_{1}\right)\right),\right. \\
\left.\chi_{\mu_{2}, P_{2}}^{2}\left(\mu_{1}+\alpha\left(\mu_{2}-\mu_{1}\right)\right)\right\} .
\end{array}
$$

If a point $y$ lies in two intervals along that line segment starting at opposite ends, and $y$ has the same $\chi^{2}$ value $d$ to each mean, then surely the ellipsoidal sets given by $\chi_{\mu_{1}, P_{1}}^{2}(x) \leq$ $d$ and $\chi_{\mu_{2}, P_{2}}^{2}(x) \leq d$ intersect because $y$ is contained in the intersection. Therefore, $d$ is an upper bound on the minimum distance such that there is an intersection, that is, $\hat{D}_{\chi^{2}}\left(\mu_{1}, P_{1} ; \mu_{2}, P_{2}\right) \geq D_{\chi^{2}}\left(\mu_{1}, P_{1} ; \mu_{2}, P_{2}\right)$. The upper bound is computed by simply solving a quadratic equation, which 


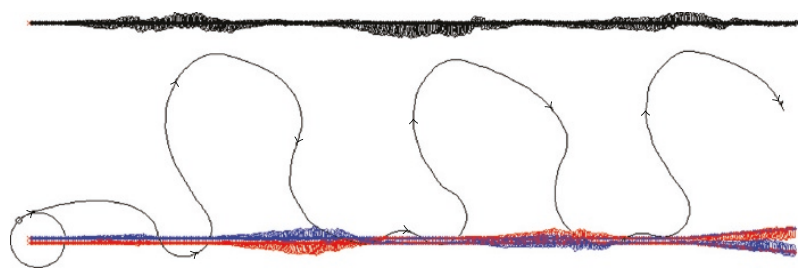

FIgure 14: $\operatorname{Tr} P$ objective in ambiguity scenario.

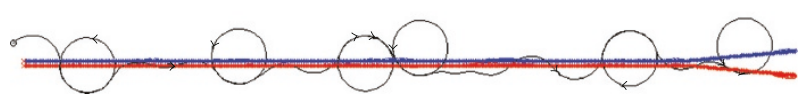

FIGURE 15: $\operatorname{Tr} P+\gamma / \widehat{D}_{\chi^{2}}$ objective in ambiguity scenario.

determines the $\alpha \in[0,1]$ such that the two $\chi^{2}$ values in (45) are equal.

7.2. Benefits of Ambiguity Objective. Using the method from Section 5, a sensor tracking two targets will try to stay near to both targets, indeed between them if possible, thereby minimizing ambiguity even without an explicit measure of ambiguity in the objective function. Thus to demonstrate the effect of a planner that deliberately seeks to minimize ambiguity requires a scenario in which at least one sensor is assigned to track at least three targets on its own.

The scenario depicted in Figures 14 and 15 demonstrates a genuine tradeoff that has to be made by the planner. Two of the targets (red and blue) are traveling very close to each other. The third (black) target is far away from the other two. If the sensor stays near the two bottom targets then it has a good chance of maintaining a clear picture of which is which, but its estimate of the top target's state remains at a consistently poor quality. If the sensor "weaves" between the top and bottom targets then it can maintain a more balanced level of estimated error amongst the targets, but it is much more likely to confuse the identity of the bottom two. Recall from Section 5.2 that weaving optimizes the mean squared tracking error objective when tracking targets that are distant from each other. With the same mean-squarederror objective function (approximated by $\operatorname{Tr} P$ ), the same behavior occurs in this scenario (Figure 14) except more time is spent in the lower region with the two closely spaced targets. By adding a term proportional to $1 / \widehat{D}_{\chi^{2}}$ to the cost, a penalty is placed on ambiguity of track states, and as seen in Figure 15, the result is that the sensor stays near the two bottom targets.

The outcomes shown in Figures 14 and 15 are in fact representative of the behavior of the two different objective functions over multiple Monte Carlo runs. Figure 16 plots the cumulative distribution of the fraction of incorrect data associations over the course of each of 5000 Monte Carlo simulations. The weaving behavior produced by the trace

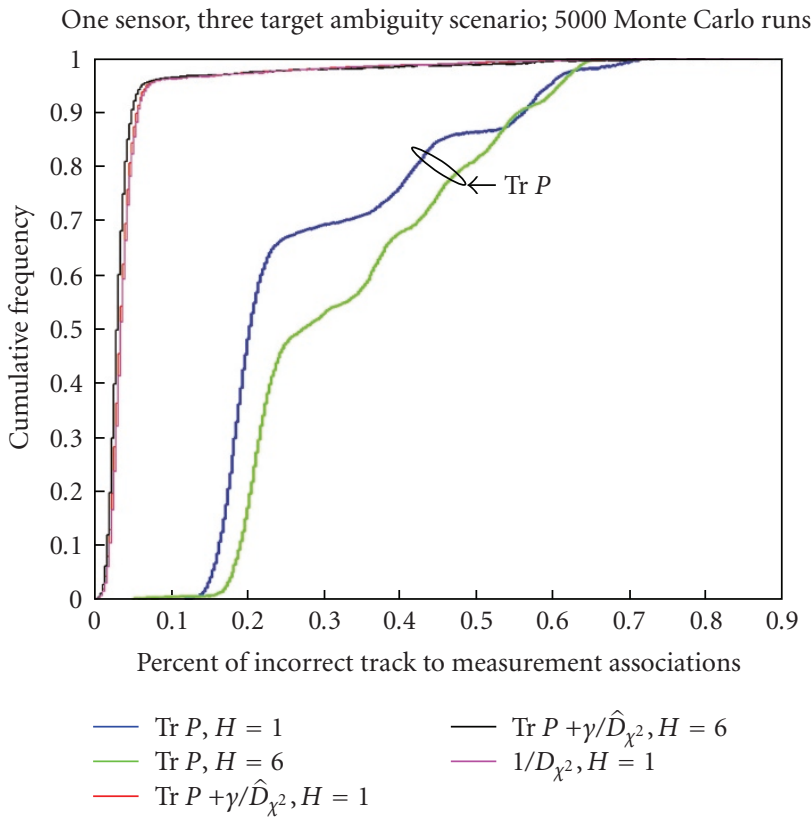

FIGURE 16: CDF of data association error rate in ambiguity scenario.

TABLE 1: Frequency of correct track association at end of scenario.

\begin{tabular}{lcc}
\hline Objective & $H$ & \% Correct ID \\
\hline $\operatorname{Tr} P$ & 1 & $70.14 \%$ \\
$\operatorname{Tr} P$ & 6 & $58.58 \%$ \\
$\operatorname{Tr} P+\gamma / \widehat{D}_{\chi^{2}}$ & 1 & $97.04 \%$ \\
$\operatorname{Tr} P+\gamma / \widehat{D}_{\chi^{2}}$ & 6 & $97.02 \%$ \\
$1 / D_{\chi^{2}}$ & 1 & $97.20 \%$ \\
\hline
\end{tabular}

objective clearly results in a higher proportion of association errors. However, as mentioned in Section 7.1, individual track to measurement associations are not our concern per se, but rather the correctness of the final track to truth association after the targets separate. Table 1 summarizes how frequently tracks are assigned to the correct target ID at the end of the scenario. Again, the benefit of including $D_{\chi^{2}}$ or $\hat{D}_{\chi^{2}}$ for ambiguity avoidance is clear. (Note that in the presence of complete ambiguity between the two targets on the bottom, we could guess the correct target ID by a coin flip and expect $50 \%$ accuracy.)

While the above results demonstrate the success of our ambiguity objective in accomplishing what it was explicitly designed for, one additional positive outcome from this scenario may be surprising at first. The objective functions that include ambiguity tend to produce a better overall mean-squared-tracking error than the trace objective alone, as seen in Figure 17. The reason is the latter equality in (21) assumes correct data association. In other words, in the presence of ambiguity, the trace of the position covariance no longer represents the mean-squared-track error relative to truth. As such, we can view the term $1 / D_{\chi^{2}}$ as a heuristic ECTG-the term plays a similar role as the ECTG term 


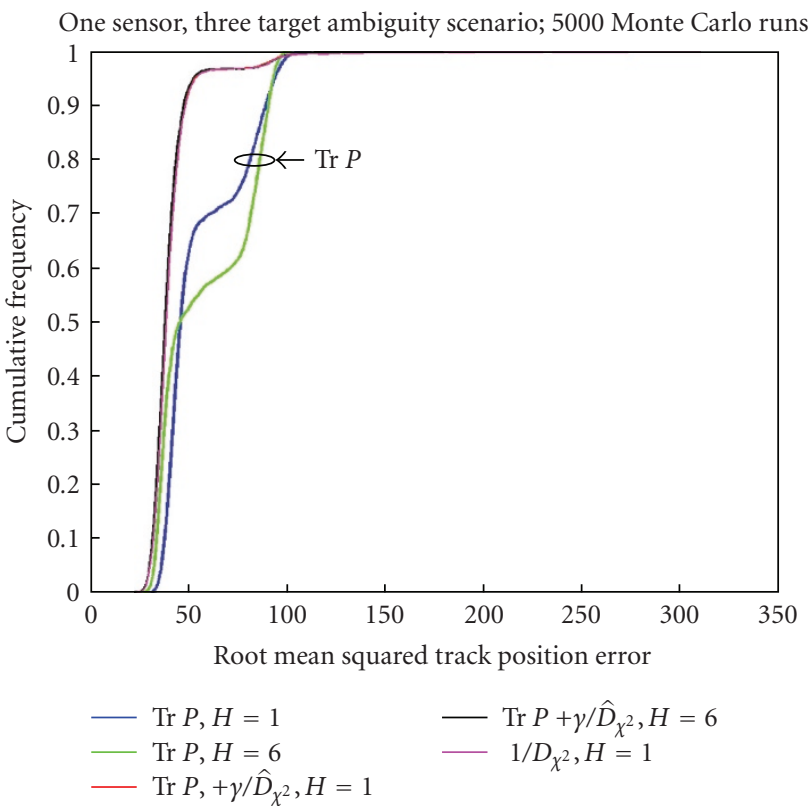

FIGURE 17: CDF of RMS track error in ambiguity scenario.

in Section 6 and contributes to improvement in the overall tracking performance.

The histogram in Figure 18 shows a clear bimodal distribution when using the trace objective, apparently corresponding to the cases where a track swap does or does not occur, respectively. Although the trace objective produces good tracking performance when no track swap occurs, the significant second mode leads to a poor average performance result. In contrast, Figure 19 shows that when the objective includes ambiguity, the mean-squared error is heavily distributed around a single mode with a very low weight on the second mode (even with the ambiguity objective the sensor occasionally produces a track swap because constraints on the UAV motion prohibit it from remaining in place between the two targets).

\section{Conclusion}

Our main contribution in this paper is a demonstration of the effectiveness of the POMDP formalism as basis for designing a solution to a complex resource management problem. The application of ideas from POMDP theory is not straightforward because approximations must be made in order to develop a practical solution. Nevertheless, by grounding the design approach in the principles of POMDP, we can preserve the key advantages of the theoretical framework, namely the flexibility to handle complex models and objectives, and the lookahead nature of the solution.

We have illustrated both of those advantages in the UAV guidance examples presented here. These simplified examples were designed to highlight some of the central issues involved in the practical application of POMDPbased design. They identified the benefit of a nonmyopic policy, the crucial importance of an approximate ECTG

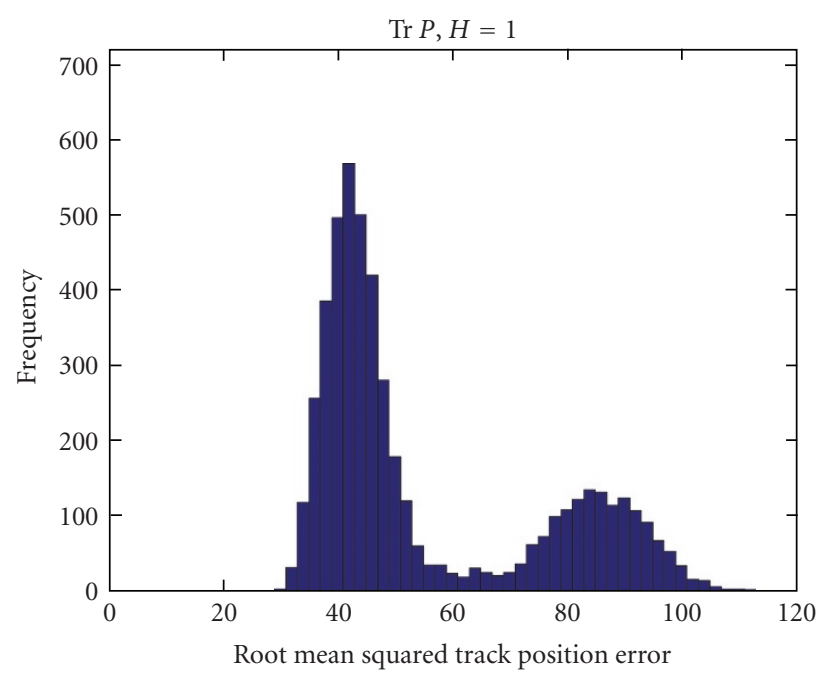

FIgURE 18: Histogram of RMS track error, Tr $P$ objective, $H=1$.

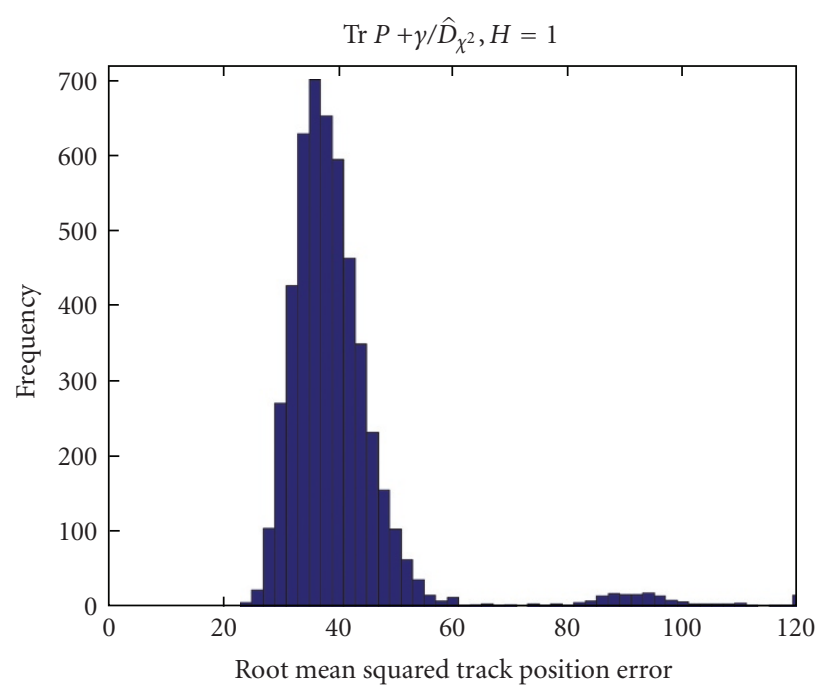

FIgURE 19: Histogram of RMS track error, Tr $P+\gamma / \widehat{D}_{\chi^{2}}$ objective, $H=1$.

term in the objective, the structured roles that heuristics can play in the algorithm (e.g., adaptive horizon length, search initialization), and the ability to change the objective without major redesign.

We have also presented a new approximation method called nominal belief-state optimization (NBO) which is particularly well suited to the tracking application considered here, because under standard assumptions the expected cost can be computed analytically. As NBO is a special case of hindsight optimization and foresight optimization, a design based on NBO is easily extended to these more computationally expensive methods if more accurate representation of the randomness of the problem is required.

As our main goal in this paper is to illustrate some of the practical issues involved in applying the POMDP-based design approach, the actual guidance system developed here 
is not meant to be taken as the best design we could achieve. There are many directions in which the algorithm could be improved, including:

(i) a more accurate MDO approximation;

(ii) a more global search for the optimal action plan;

(iii) an adaptive weight on the ECTG term (which currently requires some tuning);

(iv) a different parameterization of the action space that allows for longer planning horizons while limiting the growth of the search space;

(v) a limited use of Monte Carlo methods to explore alternative futures other than the nominal belief-state sequence.

The conclusion we wish to emphasize is that the principled framework of a POMDP-based design provides an understanding of where approximations are applied, leading to avenues of performance improvement (such as the ones listed above) as more computational resources become available.

\section{Acknowledgments}

This work was supported by AFOSR Grant no. FA9550-071-0360 and NSF Grant no. ECCS-0700559.

\section{References}

[1] B. Grocholsky, J. Keller, V. Kumar, and G. Pappas, "Cooperative air and ground surveillance," IEEE Robotics \& Automation Magazine, vol. 13, no. 3, pp. 16-26, 2006.

[2] R. A. Wise and R. T. Rysdyk, "UAV coordination for autonomous target tracking," in Proceedings of the AIAA Guidance, Navigation, and Control Conference, pp. 3210-3231, Keystone, Colo, USA, August 2006.

[3] O. Madani, S. Hanks, and A. Condon, "On the undecidability of probabilistic planning and infinite-horizon partially observable Markov decision problems," in Proceedings of the 16th National Conference on Artificial Intelligence (AAAI '99), pp. 541-548, Orlando, Fla, USA, July 1999.

[4] S. Musick, "Defense applications," in Foundations and Applications of Sensor Management, A. O. Hero III, D. Castañon, D. Cochran, and K. Kastella, Eds., chapter 11, Springer, New York, NY, USA, 2007.

[5] A. O. Hero III, D. Castañon, D. Cochran, and K. Kastella, Eds., Foundations and Applications of Sensor Management, Springer, New York, NY, USA, 2008.

[6] S. Miranda, C. Baker, K. Woodbridge, and H. Griffiths, "Knowledge-based resource management for multifunction radar," IEEE Signal Processing Magazine, vol. 23, no. 1, pp. 6676, 2006.

[7] W. Schmaedeke and K. Kastella, "Information based sensor management and IMMKF," in Signal and Data Processing of Small Targets, vol. 3373 of Proceedings of SPIE, pp. 390-401, Orlando, Fla, USA, April 1998.

[8] B. P. Grocholsky, H. F. Durrant-Whyte, and P. W. Gibbens, "Information-theoretic approach to decentralized control of multiple autonomous flight vehicles," in Sensor Fusion and
Decentralized Control in Robotic Systems III, vol. 4196 of Proceedings of SPIE, pp. 348-359, Boston, Mass, USA, November 2000.

[9] C. M. Kreucher, A. O. Hero III, K. D. Kastella, and M. R. Morelande, "An information-based approach to sensor management in large dynamic networks," Proceedings of the IEEE, vol. 95, no. 5, pp. 978-999, 2007.

[10] D. J. Klein and K. A. Morgansen, "Controlled collective motion for trajectory tracking," in Proceedings of the American Control Conference (ACC '06), pp. 5269-5275, Minneapolis, Minn, USA, June 2006.

[11] J. Lee, R. Huang, A. Vaughn, et al., "Strategies of pathplanning for a UAV to track a ground vehicle," in Proceedings of the 2nd Annual Symposium on Autonomous Intelligent Networks and Systems (AINS '03), Menlo Park, Calif, USA, June 2003.

[12] A. Ryan, J. Tisdale, M. Godwin, et al., "Decentralized control of unmanned aerial vehicle collaborative sensing missions," in Proceedings of the American Control Conference (ACC '07), pp. 4672-4677, New York, NY, USA, July 2007.

[13] D. J. Klein, C. Matlack, and K. A. Morgansen, "Cooperative target tracking using oscillator models in three dimensions," in Proceedings of the American Control Conference (ACC '07), pp. 2569-2575, New York, NY, USA, July 2007.

[14] A. D. Ryan, H. Durrant-Whyte, and J. K. Hedrick, "Information-theoretic sensor motion control for distributed estimation," in Proceedings of the ASME International Mechanical Engineering Congress and Exposition (IMECE '07), pp. 725-734, Seattle, Wash, USA, November 2007.

[15] E. K. P. Chong, C. Kreucher, and A. O. Hero III, "POMDP approximation methods based on heuristics and simulation," in Foundations and Applications of Sensor Management, A. O. Hero III, D. Castañon, D. Cochran, and K. Kastella, Eds., chapter 8, pp. 95-120, Springer, New York, NY, USA, 2008.

[16] Y. He and E. K. P. Chong, "Sensor scheduling for target tracking in sensor networks," in Proceedings of the 43rd IEEE Conference on Decision and Control (CDC '04), vol. 1, pp. 743748, Paradise Island, Bahamas, December 2004.

[17] Y. He and E. K. P. Chong, "Sensor scheduling for target tracking: a Monte Carlo sampling approach,” Digital Signal Processing, vol. 16, no. 5, pp. 533-545, 2006.

[18] L. W. Krakow, E. K. P. Chong, K. N. Groom, J. Harrington, Y. $\mathrm{Li}$, and B. Rigdon, "Control of perimeter surveillance wireless sensor networks via partially observable Marcov decision process," in Proceedings of the 40th Annual IEEE International Carnahan Conference on Security Technology (ICCST '06), pp. 261-268, Lexington, Ky, USA, October 2006.

[19] Y. Li, L. W. Krakov, E. K. P. Chong, and K. N. Groom, "Dynamic sensor management for multisensor multitarget tracking," in Proceedings of the 40th Annual Conference on Information Sciences and Systems (CISS '07), pp. 1397-1402, Princeton, NJ, USA, March 2007.

[20] Y. Li, L. W. Krakow, E. K. P. Chong, and K. N. Groom, "Approximate stochastic dynamic programming for sensor scheduling to track multiple targets," Digital Signal Processing. In press.

[21] S. Thrun, W. Burgard, and D. Fox, Probabilistic Robotics, MIT Press, Cambridge, Mass, USA, 2005.

[22] S. Thrun, "Monte Carlo POMDPs," in Advances in Neural Information Processing Systems 12, S. Solla, T. Leen, and K.R. Müller, Eds., pp. 1064-1070, MIT Press, Cambridge, Mass, USA, 2000. 
[23] J. M. Porta, N. Vlassis, M. T. J. Spaan, and P. Poupart, "Pointbased value iteration for continuous POMDPs," Journal of Machine Learning Research, vol. 7, pp. 2329-2367, 2006.

[24] S. Ross, B. Chaib-draa, and J. Pineau, "Bayesian reinforcement learning in continuous POMDPs with application to robot navigation," in Proceedings of IEEE International Conference on Robotics and Automation (ICRA '08), pp. 2845-2851, Pasadena, Calif, USA, May 2008.

[25] D. P. Bertsekas, Dynamic Programming and Optimal Control, vol. 2, Athena Scientific, Belmont, Mass, USA, 3rd edition, 2007.

[26] C. Boutilier, R. Dearden, and M. Goldszmidt, "Stochastic dynamic programming with factored representations," Artificial Intelligence, vol. 121, no. 1-2, pp. 49-107, 2000.

[27] S. Ross, J. Pineau, S. Paquet, and B. Chaib-draa, "Online planning algorithms for POMDPs," Journal of Artificial Intelligence Research, vol. 32, pp. 663-704, 2008.

[28] C. Kreucher, A. O. Hero III, K. Kastella, and D. Chang, "Efficient methods of non-myopic sensor management for multitarget tracking," in Proceedings of the 43rd IEEE Conference on Decision and Control (CDC '04), vol. 1, pp. 722-727, Paradise Island, Bahamas, December 2004.

[29] D. P. Bertsekas and J. N. Tsitsiklis, Neuro-Dynamic Programming, Athena Scientific, Belmont, Mass, USA, 1996.

[30] R. S. Sutton and A. G. Barto, Reinforcement Learning: An Introduction, MIT Press, Cambridge, Mass, USA, 1998.

[31] D. P. Bertsekas and D. A. Castañon, "Rollout algorithms for stochastic scheduling problems," Journal of Heuristics, vol. 5, no. 1, pp. 89-108, 1999.

[32] E. K. P. Chong, R. L. Givan, and H. S. Chang, "A framework for simulation-based network control via hindsight optimization," in Proceedings of the 39th IEEE Conference on Decision and Control (CDC'00), vol. 2, pp. 1433-1438, Sydney, Australia, December 2000.

[33] G. Wu, E. K.P. Chong, and R. Givan, "Burst-level congestion control using hindsight optimization," IEEE Transactions on Automatic Control, vol. 47, no. 6, pp. 979-991, 2002.

[34] L. D. Stone, C. A. Barlow, and T. L. Corwin, Bayesian Multiple Target Tracking, Artech House, Boston, Mass, USA, 1999.

[35] S. Gadaleta, S. Herman, S. Miller, et al., "Short-term ambiguity assessment to augment tracking data association information," in Proceedings of the 8th International Conference on Information Fusion (ICIF '05), vol. 1, pp. 691-698, Philadelphia, Pa, USA, July 2005.

[36] Y. Bar-Shalom and T. Fortmann, Tracking and Data Association, Academic Press, San Diego, Calif, USA, 1988.

[37] S. Ray, Distance-based model-selection with application to the analysis of gene expression data, Ph. D. thesis, Department of Statistics, Pennsylvania State University, University Park, Pa, USA, 2003.

[38] S. Boyd and L. Vandenberghe, Convex Optimization, Cambridge University Press, New York, NY, USA, 2004. 